\title{
Beliefs and Voting Decisions: A Test of the Pivotal Voter Model
}

\author{
John Duffy $^{\mathrm{a}}$ and Margit Tavits ${ }^{\mathrm{b}}$
}

This Draft: May 10, 2006

\begin{abstract}
We report results from a laboratory experiment that provides the first direct test of the pivotal voter model. This model predicts that voters will rationally choose to vote only if their expected benefit from voting outweighs the cost. The expected benefit calculation involves the use of the voter's subjective probability that s/he will be pivotal to the election outcome; this probability is typically unobservable. In one of our experimental treatments we elicit these subjective probabilities using a proper scoring rule that induces truthful revelation of beliefs. The cost of voting and the payoff to the election winner are known constants, so the subjective probabilities allow us to directly test the pivotal voter model. We find only weak support for the model. While a higher subjective probability of being pivotal does increase the likelihood that an individual chooses to vote, the decisiveness probability thresholds used by subjects are not as crisp as the theory would predict. We do find that individuals learn over time to adjust their probabilities of being pivotal so that they are more consistent with the historical frequency of decisiveness. In a second treatment, we eliminate the elicitation of decisiveness probabilities and find little change in the voting behavior of subjects; we conclude from this second treatment that our belief elicitation procedure does not alter the manner in which individuals make voting decisions and therefore provides a reasonable direct test of the pivotal voter model.
\end{abstract}

\footnotetext{
${ }^{a}$ Department of Economics, University of Pittsburgh, Pittsburgh, PA 15260, email: jduffy@pitt.edu

${ }^{\mathrm{b}}$ Department of Political Science, University of Missouri, Columbia, MO 65211, and Nuffield College, University of Oxford, OX1 1NF, email: tavitsm@missouri.edu
} 


\section{Introduction}

Why do people vote? While many theories have been offered (for a survey, see e.g., Dhillon and Peralta 2002), the simplest and most widely used framework is the pivotal voter model (Ledyard 1984, Palfrey and Rosenthal 1983, 1985; see also Downs 1957, Tullock 1967). This model asserts that voters have only instrumental concerns - their motivation is to affect the outcome of the election as opposed to non-instrumental motivations, e.g. warm-glow altruism and that in making the decision to vote they are rational, self-interested expected payoff maximizers. In particular, people vote if the expected benefit of voting is greater than their cost of voting.

Despite the widespread appeal of this model and its position as the most extensively studied and debated theory in political science (Green and Shapiro 1994: 47-48), there are few empirical studies examining its assumptions and predictions. Field data can usually provide only weak tests of the model as they pose challenge to measurement and provide little control over extraneous factors (see Levine and Palfrey 2005). Among the difficulties are the unobservability of voters' costs of voting, benefits from an election victory and their beliefs as to whether they will be pivotal to the election outcome - all of which play a critical role in the theory (Green and Shapiro 1994: 47-71).

Undoubtedly, the greatest controversy surrounds the measurement and relevance of the probability of any voter being pivotal - the trademark of the rational choice theory of turnout (Aldrich 1993, Foster 1984, Green and Shapiro 1994: 47-71). Various proxies have been used to measure pivotalness, such as the expected or perceived closeness of the election (Blais and Young 1997, Ferejohn and Fiorina 1975, Foster 1984; see also Matsusaka and Palda 1993 for a review), and the size of the electorate (Hansen, Palfrey and Rosenthal 1987, see also Bendor, 
Diermeier and Ting 2003: 274-5). However, all of these proxies have been criticized as being a "far cry" from the actual concept of pivotalness (Aldrich 1993: 259, Cyr 1975: 25, Green and Shapiro 1994: 54-55, Shachar and Nalebuff 1999). Thus, the tests based on these proxies can hardly be considered as tests of the pivotal voter model (Merlo 2005). ${ }^{1}$ Whether and how pivotalness factors into an individual's turnout calculation remains largely a puzzle and so does the performance of the pivotal voter model.

An alternative to working with field data is to conduct laboratory experiments with paid human subjects and that is the approach we take in this paper. In the laboratory we can control both the cost of voting and the payoff to the party that wins. Using neutral language and anonymous interaction we can minimize other factors that might affect voting decisions, such as the fulfillment of "civic duty" or the avoidance of peer sanctions for non-participation. Most importantly, we can elicit the beliefs that subjects have regarding whether their voting decision will be pivotal or not using a proper scoring rule that incentivizes truthful revelation of subjects' beliefs. As these beliefs are fundamental to the pivotal voter model, it is important that we acquire and examine data on beliefs so as to carefully assess the extent to which agents form correct beliefs and appropriately condition their behavior on those beliefs.

The advantages of using the laboratory to test the pivotal voter model have not gone unnoticed. Several prior experimental studies have tested various aspects of the pivotal voter model, including the implications of different voting rules (plurality, proportional), (Schram and Sonnemans (1996a)), communication, group identity and individual characteristics such as the student's university major (Schram and Sonnemans (1996b)) various comparative static

\footnotetext{
${ }^{1}$ Coate, Conlin and Moro (2004) test the pivotal voter model by looking at turnout in local Texas elections and considering closeness as a measure of pivotalness. However, as above, this does not provide a direct test of the model. See also Battaglini, Morton and Palfrey (2005: 21) on how simple tests of the effect of closeness on turnout are not nuanced enough as tests of the pivotal voter model.
} 
predictions such as how variations in electorate sizes impact on voting decisions (Levine and Palfrey (2005)) and the effects of asymmetric information (Battaglini, Morton and Palfrey (2005)). However, none of these prior studies have examined the question of subjects' subjective beliefs as to the pivotalness of their decision to vote or not, a question that lies at the heart of the pivotal voter model.

In this paper, we adopt the neutral language participation game design of Schram and Sonneman (1996ab), and add to it a belief elicitation stage that precedes the voting stage. In the belief elicitation stage, we ask subjects to state a subjective probability as to whether their own decision to vote or not will be decisive for the election outcome. We carefully explain to subjects what it means for their voting decision to be decisive. We incentivise truthful revelation of individual beliefs using a proper scoring rule and subject earnings are determined in small part by the ex post accordance of their beliefs with election outcomes. ${ }^{2}$ In addition, we are able to study whether subjects learn over time to form correct beliefs with regard to their pivotalness in the finitely repeated election game. In sum, the study provides the first direct test of the pivotal voter model.

We find that the average participation rates are consistent with the theoretical prediction suggesting that the theoretical model works well on the aggregate level. However, or main interest is on the individual level. Here, our most important finding is that there is some weak evidence that subjects condition their voting decisions on their subjective beliefs in the manner prescribed by the pivotal voter model. Specifically we find that subjects are more likely to vote the higher are their subjective beliefs, but that the crisp threshold prediction of the theory is not

\footnotetext{
${ }^{2}$ Several other experimental studies have sought to elicit subjects' subjective beliefs in environments other than the voting game that we examine. These studies include McKelvey and Page (1990), Offerman et al. (1996), Croson (2000), Nyarko and Schotter (2002), Rutström and Wilcox (2004) and Costa-Gomes and Weizsäcker (2005). The evidence from these studies regarding the impact of belief elicitation procedures on subject behavior is mixed. For this reason, we report data from our own control treatment without belief elicitation for comparison purposes.
} 
supported. We further find evidence that individuals learn over time to adjust their subjective probabilities of being pivotal in response to histories of voting outcomes in the direction of the true ex-post frequency. When we eliminate the elicitation of decisiveness probabilities we find little change in the voting behavior of subjects, leading us to conclude that our belief elicitation procedure does not alter the manner in which individuals make voting decisions.

\section{Pivotal Voter Model}

We consider the complete information participation game approach to modeling voting pursued by Palfrey and Rosenthal (1983). Specifically there are two teams of players of size $M$ and $N$ and all team members have a choice between two actions, vote (participation) or don't vote (abstention/nonparticipation). The cost of voting $c \in(0,1)$ is assumed to be the same for all agents, as is the benefit $B>0$ to each member of the winning team; each member of the losing team earns zero. The utility function is assumed to be linear, as is standard in the literature. Specifically, letting $p$ denote the probability of casting a pivotal vote, the net return to voting, $R=p B-c$. Note that we abstract away from any fixed benefits to voting, e.g., the utility one gets from a "civic duty" to vote or from the avoidance of sanctions from not voting; our neutral language experimental design makes such concerns unimportant. Normalizing $B=1$, it follows that players will rationally choose to vote whenever $p \geq c$, and will rationally chose to abstain otherwise.

The rule used to determine the outcome of voting is simple plurality. As for ties, we flipped a coin in advance of each election to determine which team would win in the event of a tie; the pre-announcement of the winner in the even of a tie aids in assessments of pivotalness (as 
described later). Given the pre-announcement of the tie-breaking rule, the setting corresponds to the "status quo" rule where there is a default winner in the event of a tie.

For our setting with $M=N>0$ and the status quo rule, it follows from Palfrey and Rosenthal (1983) that there are no pure strategy equilibria. There may exist quasi-symmetric, totally mixed strategy equilibria where each member of the group that does not win a tie chooses to vote with probability $q$, defined implicitly by

$$
\left(\begin{array}{c}
M+N-1 \\
N
\end{array}\right) q^{N}(1-q)^{M-1}=c,
$$

and members of the group that wins a tie vote with probability 1-q. As Palfrey and Rosenthal show there exist values of $c$ for which equation (1) yields either 0,1 or 2 solutions for $q$.

We chose parameters for the experiment, $M=N=10$ and $c=.18$ that are very close to the case where there is a unique, quasi-symmetric totally mixed strategy equilibrium. Our aim was to try to reduce the set of equilibria that subjects might coordinate on so as to have a more reasonable chance of predicting turnout. ${ }^{3}$ In the unique mixed strategy equilibrium with $M=N$ $=10, q=N /(N+M-1)=.53$ and $1-q=.47^{4}$ It follows that turnout in this equilibrium involves $(2 M-1) N /(N+M-1)=10$ participants out of an electorate of size 20, or a turnout rate of 50 percent. While turnout is of interest to us, the primary focus of this paper is on the consistency of subjects' beliefs with their action choices. We now turn to a description of our experimental design and main hypotheses.

\section{Experimental Design and Hypotheses}

\footnotetext{
${ }^{3}$ There may also exist asymmetric equilibria, where some agents play pure strategies while others play mixed strategies, but for simplicity we focus on symmetric strategies.

${ }^{4}$ The value of $\mathrm{c}$ needed to implement the unique mixed strategy equilibrium is .17697 . Given that the smallest increment of monetary payment is .01 , we chose to set $c=.18$. Technically speaking, for $c=.18$, there are two totally mixed strategy equilibria, $q_{1}=.514883$ and $q_{2}=.53773$, but we prefer to consider $q=.53$ as the relevant benchmark.
} 
Our experiment involves two main treatments. In the "control" treatment we did not elicit subjects' beliefs as to whether their voting decision would be pivotal while in the "beliefs" treatment, we did. We conducted 3 sessions of the control treatment and 3 sessions of the beliefs treatment. We first describe the control treatment; the beliefs treatment differs only in our elicitation of beliefs prior to voting decisions.

\section{Control Treatment}

In the control treatment, subjects were randomly assigned to one of two groups labeled X and $\mathrm{Y}$ at the start of the experimental session. We were careful to use neutral language in both treatment and avoid any context with regard to voting or elections as we did not want to cue subjects' beliefs with regard to social norms/sanction surrounding voting decisions. Subjects were told that in each "round" of the experiment (20 rounds total), they were to decide whether to purchase a "token" or not (equivalent to casting a vote or abstaining). Purchasing a token cost them $\$ 0.18$, i.e., we set the cost of voting to $c=.18$. The payoff to the winning group is $\$ 1$ and the losing group earns $\$ 0$.

The experimental instructions (available on request) made the payoffs to the winning team and the cost of buying a token public knowledge to all subjects. In addition, the instructions explained the plurality rule used to determine the winning group and the pre-announced tiebreaking outcome, which was to pick one team randomly each round to be the winning team in the event of a tie. Prior to the start of the experiment, subjects had to answer several quiz questions designed to test their understanding of the instructions. Subjects played 20 rounds of this game remaining in the same team over all rounds. ${ }^{5}$ They were paid their net earnings from all 20 rounds played.

\footnotetext{
${ }^{5}$ We considered random re-matching of subjects into the two teams, but thought that such a design might adversely affect subject learning, especially with regard to the probability that any individual subject is pivotal. On the other
} 
The timing of moves within a round was a follows. First the random determination as to which team would win a tie was announced. Second subjects were asked to decide whether or not to purchase a token. Finally, the results of the round were revealed as in the control treatment.

At the end of each round, subjects were informed of the number of members of their group of 10 who purchased a token, the number of members of the other group of 10 who purchased a token and which team won for that round. In the event of a tie, the pre-announced tie-breaking rule determined the winning group. All members of the winning group earned $\$ 1$ less the cost of purchasing a token, if they purchased a token. Similarly, all members of the losing group earned $\$ 0$ less, the cost of purchasing a token, if they purchased a token.

Notice that each round of the control treatment, subjects' net earnings consist of one of four possible payoffs: $\$ 1, \$ 0.82, \$ 0$ or $-\$ 0.18$; the latter negative payoff occurs when a subject buys a token and her team loses. To rule out the possibility that subjects finish the experiment with a net loss, we provided subjects with a $\$ 6$ show-up fee. As we only played 20 rounds of the voting game, the maximum loss possible was $20 \times-.18=\$ 3.60$ and subjects were informed that such losses would come out of their show-up fee. In practice, all subject payments (including the show-up payment) were greater than $\$ 6$ for both treatments. The average payoff for the 20 rounds played by subjects in the three control sessions was $\$ 20.55$ for a 90 minute experiment.

\section{Belief Elicitation Treatment}

The belief elicitation treatment differed from the control treatment in only one respect. Prior to deciding whether or not to buy a token, subjects were asked to report their subjective 
belief as to whether their decision to buy a token would be decisive (pivotal) or not. ${ }^{6}$ To aid subjects in formulating this belief, the conditions under which their decision to buy or not buy a token would be decisive were carefully explained in the experimental instructions. The decisiveness conditions made use of the fact that one group was randomly selected at the start of each round to be the winning group in the event of a tie.

The timing of moves within a round was as in the control treatment, but with the addition of the belief elicitation stage. Specifically, the timing was as follows. First the random determination as to which team would win a tie was announced. Second subjects then stated their subjective belief as to whether their token purchase decision would be decisive. Third subjects were asked to decide whether or not to purchase a token. Finally, the results of the round were revealed as in the control treatment. The information revealed at the end of each round included the same information that was revealed at the end of a control session, and additionally, subjects were reminded of their stated belief and whether their token purchase decision was decisive or not for the outcome of the round. The latter information was intended to provide subjects with the feedback necessary to better align their decisiveness beliefs with actual outcomes.

It is perhaps useful to quote the instructions with regard to the conditions under which an individual subject's token purchase decision is decisive:

"You are decisive under any of the following conditions.

Suppose that group $\mathrm{X}$ wins a tie.

1. If there is a tie then everyone in group $\mathrm{X}$ who bought a token is decisive.

2. If there is a tie then everyone in group $\mathrm{Y}$ who did not buy a token is decisive.

3. If group $\mathrm{X}$ loses by one token, then everyone in group $\mathrm{X}$ who did not buy a token is decisive.

4. If group $\mathrm{Y}$ wins by one token, then everyone in group $\mathrm{Y}$ who bought a token is decisive.

\footnotetext{
${ }^{6}$ For current purposes, we consider the terms "decisive" and "pivotal" as synonyms. In the instructions we referred used the term "decisive" in order to make the concept easier to understand for the subjects. As explained below, subjects were given a precise working definition of "decisive".
} 
Suppose instead that $\mathrm{Y}$ wins a tie.

1. If there is a tie then everyone in group $\mathrm{Y}$ who bought a token is decisive.

2. If there is a tie then everyone in group $\mathrm{X}$ who did not buy a token is decisive.

3. If group $\mathrm{Y}$ loses by one token, then everyone in group $\mathrm{Y}$ who did not buy a token is decisive.

4. If group $\mathrm{X}$ wins by one token, then everyone in group $\mathrm{X}$ who bought a token is decisive.

If one of the above conditions does not hold, then you are not decisive."

To make it incentive compatible for subjects to report their true beliefs regarding decisiveness, we make use of a proper scoring rule and give subjects a small payment according to the accuracy of their stated beliefs. Specifically, we make use of the quadratic scoring rule originally developed by Brier (1950) for weather forecasting but more recently adopted by many experimentalists (McKelvey and Page (1990), Offerman et al. (1996), Nyarko and Schotter (2002) among others). Suppose a subject reports the subjective probability $p$ that she will be decisive. Ex post, when the election results are determined, either she is decisive or not. Let $I_{D}$ be an indicator function that takes on the value 1 if the subject is decisive and 0 otherwise. The payoff we give to subjects for their stated belief each period is $\left.\pi(p)=.10\left[1-p-I_{D}\right)\right]^{2}$. That is, the maximum subjects can earn for a correct guess is $\$ 0.10$ and this amount diminishes quadratically as the guess deviates from the actual outcome, down to $\$ 0.00$. Theoretically, the quadratic scoring rule induces a risk-neutral agent to report her true, subjective belief with regard to the binary event, in our case, being decisive in the participation game (see, e.g., the discussion in Camerer (1995, pp. 592-3)). In setting the payoff for the decisiveness prediction, we followed Nyarko and Schotter (2002) in making this payoff small with respect to the payoff of winning an election (which was \$1). A concern is that when subjects earn payments for both their action choices and for the accuracy of their stated beliefs, they might state beliefs in a strategic fashion that provided them with insurance against election outcomes. By keeping the payment for belief accuracy small, we sought to minimize such strategic behavior. 
Aside from elicitation of beliefs before voting decisions, there were no differences between the two treatments. Subjects in the belief-elicitation treatments earned slightly more on average, $\$ 21.75$ than subjects in the control treatment, but the differences are easily accounted for by the additional payments subjects received in the former treatment for the accuracy of their beliefs.

Our main interest in this experimental design is to assess the extent to which subjects choose to vote when their decisiveness beliefs exceed the cost of voting, $p>c=.18$, and abstain from voting otherwise. We are also interested in whether subjects learn over time to adjust their beliefs toward the actual frequency of being decisive and whether our belief elicitation procedure impacts on decision-making, perhaps by making subjects think harder about the rationality of choosing to vote. We turn next to examining these main questions.

\section{Results}

We report results from six sessions -- three belief elicitation treatment sessions and three control treatment sessions. Each session involved 20 subjects who made decisions in 20 rounds. Thus, we have 1200 participation (or voting) decisions from the belief elicitation treatment and the same number from the control treatment, i.e. a total of 2400 decisions.

Aggregate results

We first consider the aggregate results. Figure 1 and Table 1 summarize turnout using 5period averages for each of the six sessions, labeled belief or no-belief sessions 1,2,3. The average turnout in rounds $1-5$ is around $50 \%$. It, thus, corresponds well with the theoretical prediction (see above). However, in all sessions, the turnout levels drop off over time.

The turnout levels and trends across treatments are similar enough to suggest that our belief elicitation procedure was not obtrusive. To be sure, turnout is slightly higher when beliefs 
were elicited: the average turnout is $45 \%$ in the belief treatment and $39 \%$ in the control treatment. Further, the turnout level drops off slightly more sharply in the control sample. However, the differences across the two treatments are not large; using a non-parametric MannWhitney test (results in the final column of Table 1), the null hypothesis of no difference in turnout rates between treatments can be rejected only in periods $15-20$ at the .05 level of significance. In one of the no-belief sessions, session 2, one group became dominant, i.e. the same group was winning in all periods. This may account lower participation rate in this session (see Figure 1). In all other sessions, including all three sessions where beliefs of being pivotal were elicited, no dominant group emerged. Additionally, the share of decisive games was very similar across treatments: in the belief sample, 14 out of the 60 games resulted in a decisive participation, while in the control treatment the ratio was 12 out of 60 . We will return to the issue of the potential obtrusiveness of the experimental treatment below. To foreshadow the conclusion, we find no significant differences in the results across treatments indicating the unobtrusiveness of the belief elicitation procedure.

\section{Individual level results}

The crucial independent variable in this study is the subjective decisiveness probability. Subjects could state a probability with an accuracy of up to three decimal places. In all sessions, "0" and ".5" were modal values, though many other values were chosen. The mean subjective probability that an individual is decisive is rather high: .33. It varies slightly by session, equal to .29 for the first and the third belief sessions and .41 for the second belief session.

Figure 2 shows frequency and cumulative frequency distributions for the subjective decisiveness probabilities by session averaged over the first and last 10 periods. As the graphs illustrate, subjects' decisiveness probabilities in the first 10 periods are spread somewhat 
uniformly over the interval $[0,1]$, as compared with the last 10 periods where the distribution is more skewed to the left of the interval. This is also reflected in the cumulative frequency distributions; the cumulative frequency distributions for periods 11-20 nearly always lie above those for periods $1-10$.

Figure 3 presents the mean decisiveness probabilities over time. The trend for the first session shows clear convergence in beliefs across subjects over time. The slope of the fitted regression line is negative and significant. Further, the standard deviation bounds around the mean narrow considerably towards the end of the series. A similar trend is less evident in the other two sessions, although in both cases the fitted slopes are negative.

On average, $63 \%$ of subjects across all sessions stated a probability of being decisive that was higher than .18 . Recall that $c=.18$; thus, the decisiveness probability of .18 serves as the theoretical cutpoint for participation. These subjective probabilities of being decisive can be compared to the actual probabilities, or the frequencies of past decisiveness. ${ }^{7}$ The actual probabilities average to about .12 across all sessions and are highest in the second session: .16. The differences in the average objective and subjective probabilities of being decisive are rather substantial. Figure 4 illustrates, by sessions, how over time the difference between objective decisiveness and the average subjective decisiveness decreases. The downward trend is especially visible in the case of the first session (solid line) where in the last two rounds the objective and average subjective probabilities are equal. This indicates that individuals learn over time to adjust their subjective probabilities of being pivotal in response to histories of voting outcomes in the direction of the true ex post frequency of turnout. The positive values of the

\footnotetext{
${ }^{7}$ These frequencies are calculated for each round but the first and for each subject by taking the average of subject's decisiveness across all previous rounds.
} 
series in Figure 4 indicate that subjects are almost without an exception overestimating the probability.

We further performed multivariate tests to estimate the effect of the subjective beliefs of being pivotal on the likelihood of buying a token. The results are presented in Table 2. Model 1 estimates the effect of the stated beliefs of being pivotal (continuous variable), while Model 2 replicates the same analysis using a dummy variable coded " 1 " for those who stated a probability of being pivotal higher than .18 in order to test the exact predictions of the theory.

Both models also include several controls. First, we control for whether the group of which the subject is a member will win in the case of a tie. This variable functions as a preelection poll announcing a lead to one candidate. The pivotal voter model predicts lower turnout for the "advantaged" group (see p. 6; Levine and Palfrey 2005). Further, since we ran several periods of "elections" and the group members stayed the same across rounds, we also control for various history effects. These include (1) whether a given subject was pivotal in the last round, (2) whether the subject bought a token in the last round, (3) whether the subject's group won last round, (4) the number of tokens bought by the subject's group in the last round, (5) the subject's earnings from the last round, and (6) whether there was a tie in the last round. We also control for the session dummies and the period number.

All models are estimated with the probit regression. The results of both Model 1 and Model 2 show a strong effect of the stated probability of being decisive on the probability of buying a token. Substantively, for Model 1, the predicted probability of buying a token is .37 when the stated probability of being pivotal is 0 (i.e. at its minimum) and .58 when it is 1 (at its maximum), holding other variables at their mean (for continuous variables) or median (for categorical variables). For Model 2, the predicted probability for buying a token is .4 when the 
stated probability of being pivotal is higher than .18 and only slightly higher $-.47-$ when it is lower than .18 , all other variables at their mean or median.

There is, thus, some evidence that the subjective probability of being pivotal plays a significant role in people's decision to participate: the higher the subjective probability the greater the likelihood of buying a token. The result is not, however, as crisp as the theory would predict: a subject probability of .18 does not function as a clear cutpoint for the decision to participate. Figure 5 further illustrates this point. The left panel of this figure shows the percentage of subjects who bought a token (chose to participate) after having stated a probability of being pivotal that was less than .18 while the right panel shows the percentage of subjects who bought a token after having stated a probability of being pivotal that was greater than or equal to .18. The figures show average percentages over periods 1-2 and 19-20 of each session. If players were playing according to the crisp cutpoint prediction of the theory, the percentages in the left panel would all be zero while those in the right panel would all be 100 . Notice however, that in 2 of the 3 belief sessions, the participation rate when $p<.18$ decreases as we move from periods 1-2 to the final periods 19-20, while in all 3 belief sessions, the participation rate increases when $\mathrm{p} \geq .18$ as we move from periods $1-2$ to the final periods $19-20$; the latter finding is strong evidence that subjects are learning to behave in a manner consistent with the theoretical cutpoint prediction.

Figure 6 plots average beliefs of being decisive over time for participants and nonparticipants. As is evident from these graphs, although the decisiveness probabilities of participants are usually higher than those of non-participants, there does not appear to be a clear average cutpoint for participation. In sum, there is only weak support for the specific prediction of the theory. Few participants use the exact deterministic cutpoint strategy predicted by the 
theory. However, there is evidence that subjects' behavior tends towards the theoretical prediction with higher subjective probabilities increasing the likelihood of participation.

In addition to the main findings, some of the variables measuring the effects of history or past behavior are also significantly related to the decision to participate. First, the period has a significant negative effect on the probability of buying a token: all else equal subjects were less likely to buy a token in later than in earlier periods. This may indicate a certain learning effect, for example in terms of cumulative disappointment in low payoffs from buying a token, or the emergence of a free rider problem (see Bendor, Diermeier and Ting 2003, Kanazawa 2000 for learning effects). This result also reflects the observation that turnout declines when democracies mature, i.e. as a result of repeated elections (Kostadinova 2003). Second, subjects are more likely to participate when they have participated before. This result reflects the argument about the "habitual voter" (Gerber, Green and Shachar 2003, Plutzer 2002) made in the previous empirical literature on turnout. Further, the extent to which one's group members participated in the pervious period is also significantly influencing individual's decision to participate: a subject is less likely to participate if the general participation rate in his or her group was high indicating the emergence of a free rider problem. Past group success or failure does not play a significant role in decisions to participate.

Models 1 and 2 also allow us to test a further implication of the theory. Recall that in the unique mixed strategy equilibrium that we focus on that the turnout probabilities for the two groups are not the same (see p. 6). Rather, we predicted an underdog effect (Levine and Palfrey 2005), where the group winning a tie should have higher turnout than the disadvantaged group. The "Group wins tie" variable in Models 1 and 2 captures this relationship. Contrary to the theory, the finding indicates that the tie breaking rule acts as a coordination device for voters, 
mobilizing (rather than de-mobilizing) them behind a "leading" candidate. Substantively, in both models, the predicted probability of buying a token is .34 for a member of the group that is announced to win a tie compared to .25 for a member of the other group. Figure 7 further illustrates this effect. It presents the average turnout in all six sessions for the advantaged and disadvantaged groups. In all sessions, the turnout is higher for the former, the difference being statistically significant (using a non-parametric Mann-Whitney test) in all but the second belief session. This finding is puzzling from the point of view of our theory, but it corroborates the bandwagon effect, which argues that voters favor a party that is doing well on the polls (McAllister and Studlar 1991). In the current context, this finding may have occurred as an artifact of our particular experimental design, i.e. the use of ex ante coin toss (or status quo) rule for breaking ties and fixed group membership. An alternative yet strategically equivalent design might declare in advance that one group always wins a tie and employ random re-matching of subjects into groups rather than fixed membership. If the bandwagon effect disappears, then it is possible to attribute the current result to the design.

In two additional models - Model 3 and 4 - presented in the last two columns of Table 2, we added the variable measuring the actual frequency of being decisive. Adding this variable does little to diminish the effect of the subjective probabilities of being decisive. Rather, the objective frequencies have no statistically significant effect on turnout and the coefficient has a wrong sign, while the effect of subjective beliefs remains still significant and in the predicted direction. This underlines the importance of subjective beliefs of being pivotal in turnout decisions and challenges the use of some objective measures of this probability when testing the pivotal voter model such as closeness of an election. As Figure 4 demonstrated, although over 
time the subjective probability of being pivotal tends towards the actual frequency, the differences can be substantial.

\section{Testing the obtrusiveness of the belief elicitation procedure}

Table 3 replicates Model 3 with data from the control treatment (Model 5) and from all sessions combined (Model 6). The goal here is to determine whether subjects behaved significantly differently when beliefs were elicited compared to the control group. Most importantly, the dummy variable differentiating between treatment and control sessions (variable name Belief elicitation) in Model 6 is not statistically significant. This allows concluding that there are no significant differences in the behavior of subjects across treatments and that our belief elicitation procedure was not obtrusive in terms of making subjects more aware of the rationality of participating.

Furthermore, Model 5 produces roughly similar results as Model 3 in Table 2. As above, we find that the tie breaking rule influences turnout, prior participation increases while high level of group participation depresses the likelihood of current participation, and turnout decreases significantly over time. Further, as above, the effect of the actual probability of decisiveness falls short of the conventional level of statistical significance, although it is significant at .10 level. The actual probability of decisiveness most likely acts as a weak proxy for the subjective probabilities. These similarities across treatments further suggest that the belief elicitation was unobtrusive in terms of influencing subjects' decision making.

There are also some differences however: both prior earnings and history of ties significantly and positively influence turnout. These history effects are likely to influence one's subjective probability of being pivotal, and as such, the relationships may reflect some of the effects otherwise captured by the subjective beliefs. 


\section{Conclusions}

The pivotal voter model that builds on Downs' (1957) rational choice theory of turnout is the most intuitive, yet also the most controversial formal theory in political science. Empirical tests of the theory to date have mostly relied on proxies or only been partial. The concept around which much of the controversy revolves - the probability of being pivotal - has received the least empirical attention. Indeed, pivotalness is simply inferred from the closeness of the election, and the individual level calculus of voting is never unpacked.

This study has provided the first direct test of the pivotal voter model, with a specific focus on whether and how voters' beliefs of being pivotal factor into their voting decisions. We used a laboratory setting that allows manipulating model parameters and elicited voters' beliefs about pivotalness. Importantly, we determined that the belief elicitation procedure was unobtrusive - it did not prompt subjects to alter their turnout behavior. On the aggregate level, we find support for the pivotal voter model with aggregate turnout rates corresponding well with the theoretical predictions.

The main focus of this study, however, is on the individual level behavior, where we find mixed support for the theory. Most importantly, we find evidence that those who believe that their participation will be pivotal for the outcome of a game (an election), are more likely to participate. This relationship is strong and robust, but it is not deterministic. That is, we find that voters do not use the exact deterministic cutpoint strategy of participation predicted by the theory. Those who state a probability of being pivotal higher than the cost of voting participate with a higher likelihood than those who state a lower probability, but the relationship is not deterministic. Many voters stating much higher probabilities of being pivotal still chose to 
abstain and those who stated a lower probability chose to participate, i.e. there is a stochastic element in voter choice.

Additionally, we find that the subjective beliefs are more important for the participation (turnout) decision than the actual frequencies of being decisive. Indeed, the subjective probabilities tend to be considerably higher than the actual ones - undermining the common view that closeness is a useful proxy for pivotalness in testing rational models of turnout. Yet, we also find that beliefs become more accurate over time, indicating a considerable learning effect in the turnout decision as proposed by some recent theoretical and empirical studies (Bendor, Diermeier and Ting 2003, Kanazawa 2000). We also find evidence that people use the preannounced tie breaking rule as a decision guide: they are more likely to participate if their group is announced to win a tie. This result contradicts our theoretical prediction but confirms the bandwagon effect induced by election polls.

\section{References}

Aldrich, John, H. 1993. "Rational Choice and Turnout." American Journal of Political Science 37(1): 246-278.

Bendor, Jonathan, Daniel Diermeier, and Michael Ting. 2003. "A Behavioral Model of Turnout." American Political Science Review 97(2): 261-280.

Blais, André and Robert Young. 1999. "Why Do People Vote? An Experiment in Rationality." Public Choice 99: 39-55.

Brier, Glenn W. 1950. "Verification of Forecasts Expressed in Terms of Probability," Monthly Weather Review 78:1-3. 
Camerer, Colin. 1995. "Individual Decision Making” in John H. Kagel and Alvin E. Roth, Eds. The Handbook of Experimental Economics, Princeton: Princeton University Press.

Costa-Gomes, Miguel A. and Georg Weizsäcker. 2005. "Stated Beliefs and Play in Normal Form Games," working paper, London School of Economics and Political Science.

Croson, Rachel T.A. 2000. "Thinking Like a Game Theorist: Factors Affecting the Frequency of Equilibrium Play," Journal of Economic Behavior and Organization 41: 299-314.

Cyr, A. Bruce. 1975. “The Calculus of Voting Reconsidered.” Public Opinion Quarterly 39(1): $19-38$.

Dhillon, Amrita and Susana Peralta. 2002 "Economic Theories of Voter Turnout" Economic Journal 112: F332-F352.

Ferejohn, John and Morris P. Fiorina. 1975. "Closeness Counts Only in Horseshoes and Dancing." American Political Science Review 69: 920-925.

Foster, Carroll B. 1984. “The Performance of Rational Voter Models in Recent Presidential Elections." American Political Science Review 78(3): 678-690.

Gerber, Alan S., Donald P. Green and Ron Shachar. 2003. "Voting May be Habit-Forming: Evidence from a Randomized Field Experiment." American Journal of Political Science 47(3): 540-550.

Green, Donald P. and Ian Shapiro. 1994. Pathologies of Rational Choice Theory. New Haven: Yale University Press.

Hansen, Steven, Thomas Palfrey and Howard Rosenthal. 1987. "The Relationship between Constituency Size and Turnout: Using Game Theory to Estimate the Cost of Voting." Public Choice 52: 15-33. 
Kanazawa, Satoshi. 2000. "A New Solution to the Collective Action Problem: The Paradox of Voter Turnout." American Sociological Review 65(3): 433-442.

Kostadinova, Tatiana. 2003. "Voter Turnout Dynamics in Post-Communist Europe.” European Journal of Political Research 42: 741-759.

Ledyard, John O. 1984. “The Pure Theory of Large Two-Candidate Elections” Public Choice 52(1): 15-33.

Levine, David K. and Thomas R. Palfrey. 2005. "The Paradox of Voter Participation? A Laboratory Study,” working paper, UCLA and Princeton University.

McAllister, Ian and Donley T. Studlar. 1991. "Bandwagon, Underdog, or Projection? Opinion Polls and Electoral Choice in Britain, 1979-1987.” Journal of Politics 53(3): 720-741.

McKelvey, Richard D. and Talbot Page. 1990. "Public and Private Information: An Experimental Study of Information Pooling,” Econometrica, 58: 1321-39

Matsusaka, John G. and Filip Palda. 1993. "The Downsian Voter Meets the Ecological Fallacy." Public Choice 77: 855-878.

Merlo, Antonio. 2005. "Whither Political Economy? Theories, Facts and Issues." Paper presented at the World Congress of the Econometric Society, London, August 19-24.

Nyarko, Yaw and Andrew Schotter. 2002. “An Experimental Study of Belief Learning Using Elicited Beliefs," Econometrica, 70: 971-1005.

Offerman, Theo, Joep Sonnemans and Arthur Schram. 2001. "Expectation Formation in StepLevel Public Good Games,” Economic Inquiry 2:250-69

Palfrey, Thomas R. and Howard Rosenthal. 1983. “A Strategic Calculus of Voting.” Public Choice 41: 7-53. 
Palfrey, Thomas R. and Howard Rosenthal. 1985. "Voter Participation and Strategic Uncertainty." American Political Science Review 79(1): 62-78.

Palfrey, Thomas R. and Howard Rosenthal. 1988. "Private Incentives in Social Dilemmas: The Effects of Incomplete Information and Altruism” Journal of Public Economics 35: 309332.

Plutzer, Eric. 2002. "Becoming a Habitual Voter: Inertia, Resources, and Growth in Young Adulthood.” American Political Science Review 96(1): 41-56.

Rutström, E. Elisabet and Nathaniel T. Wilcox 2004. "Learning and Belief Elicitation: Observer Effects," working paper, University of Houston.

Schram, Arthur and Joep Sonnemans. 1996a. "Voter Turnout as a Participation Game: An Experimental Investigation.” International Journal of Game Theory 25: 385-406.

Schram, Arthur and Joep Sonnemans. 1996b. "Why People Vote: Experimental Evidence.” Journal of Economic Psychology 17: 417-442.

Shachar, Ron and Barry Nalebuff. 1999. "Follow the Leader: Theory and Evidence on Political Opinion." American Economic Review 89(3): 525-547. 
Figure 1: Average Turnout

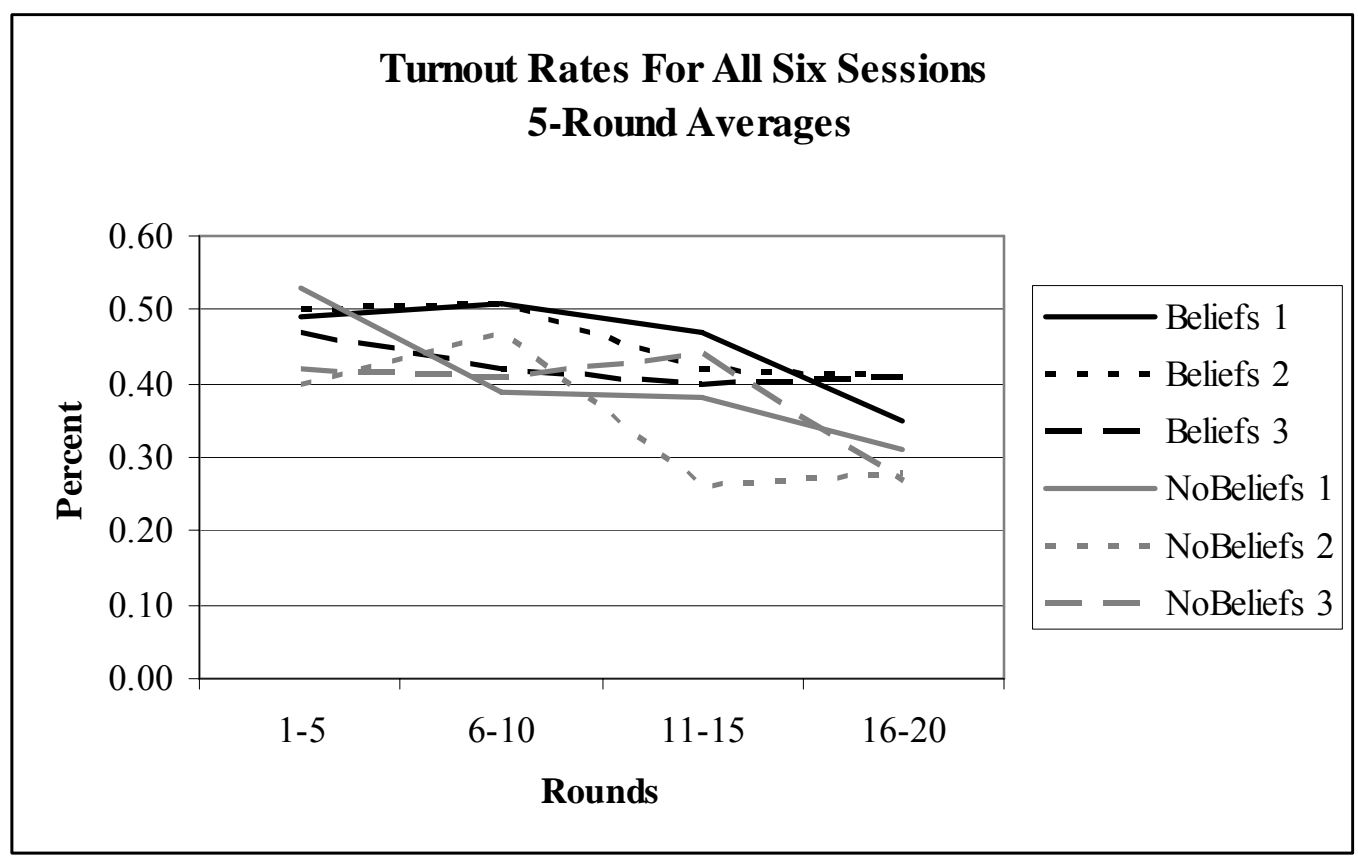


Figure 2: Frequency (left column) and cumulative frequency distribution (right column) of subjective decisiveness probabilities by session, averages over period 1-10, 11-20.
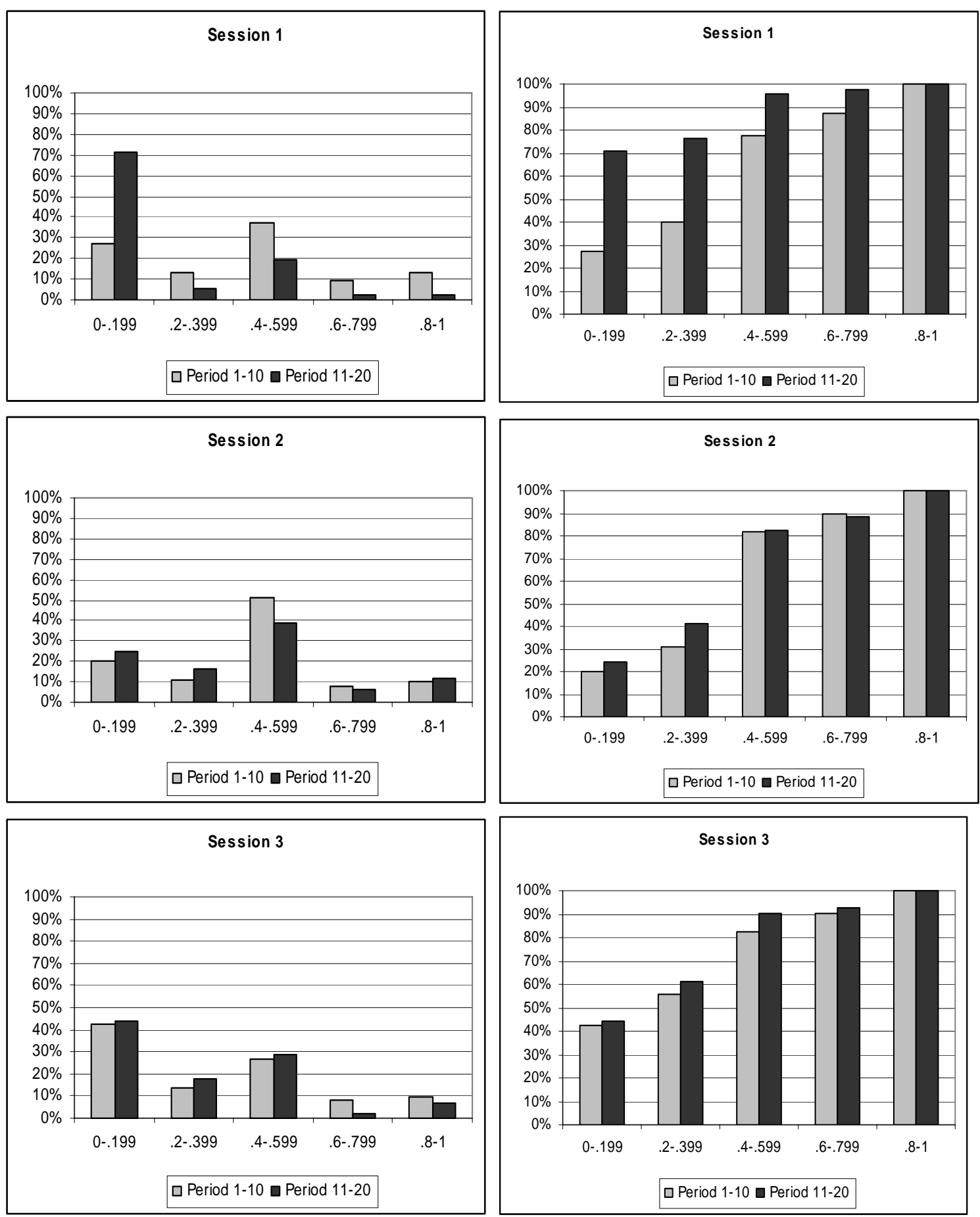
Figure 3: Mean decisiveness probabilities over time by session
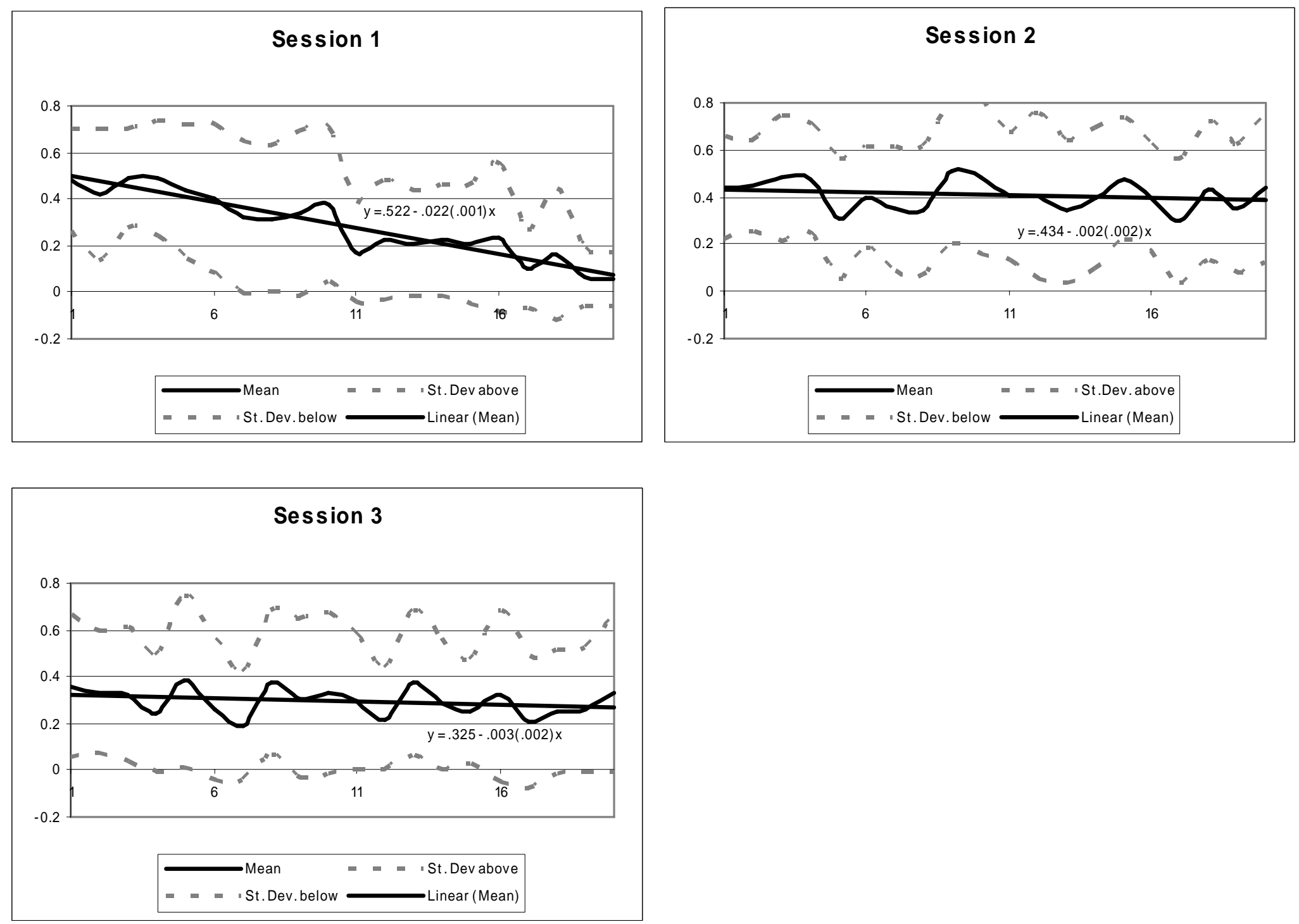
Figure 4: The difference in the subjective probability and actual frequency of being decisive

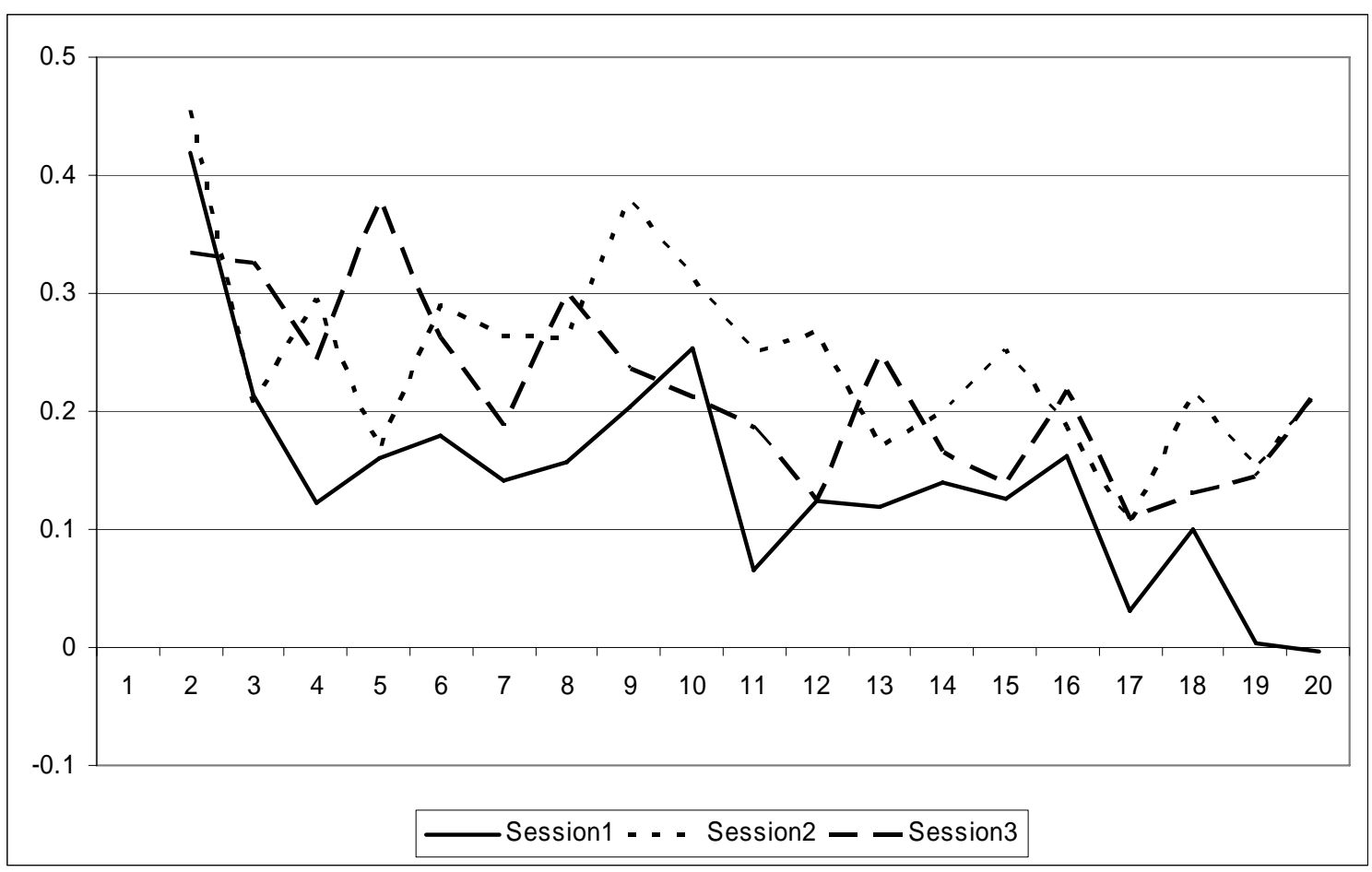


Figure 5: Participation rate by decisiveness probability
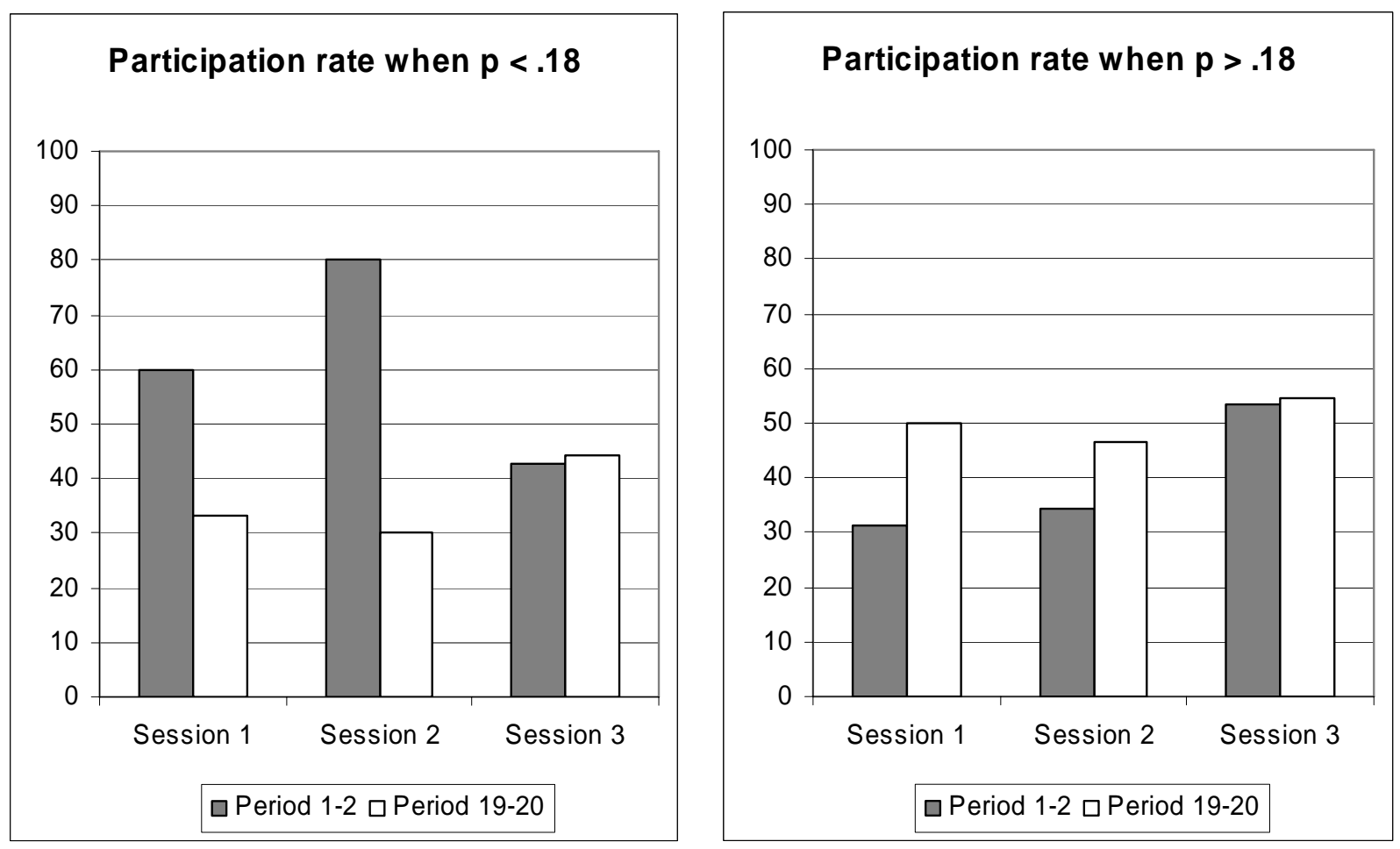
Figure 6: Average beliefs of being decisive over time for participants and non-participants
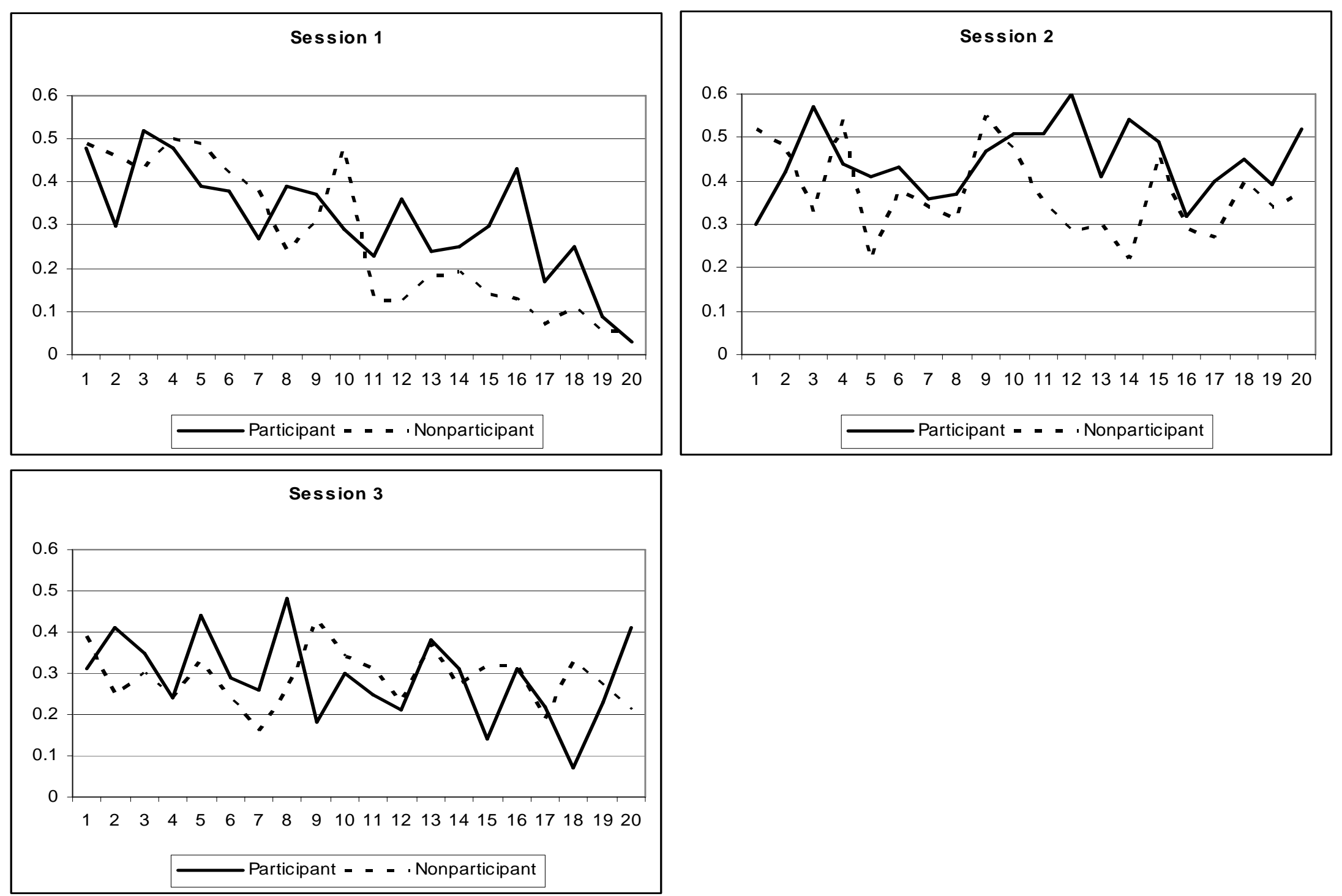
Figure 7: Turnout in advantaged and disadvantaged group

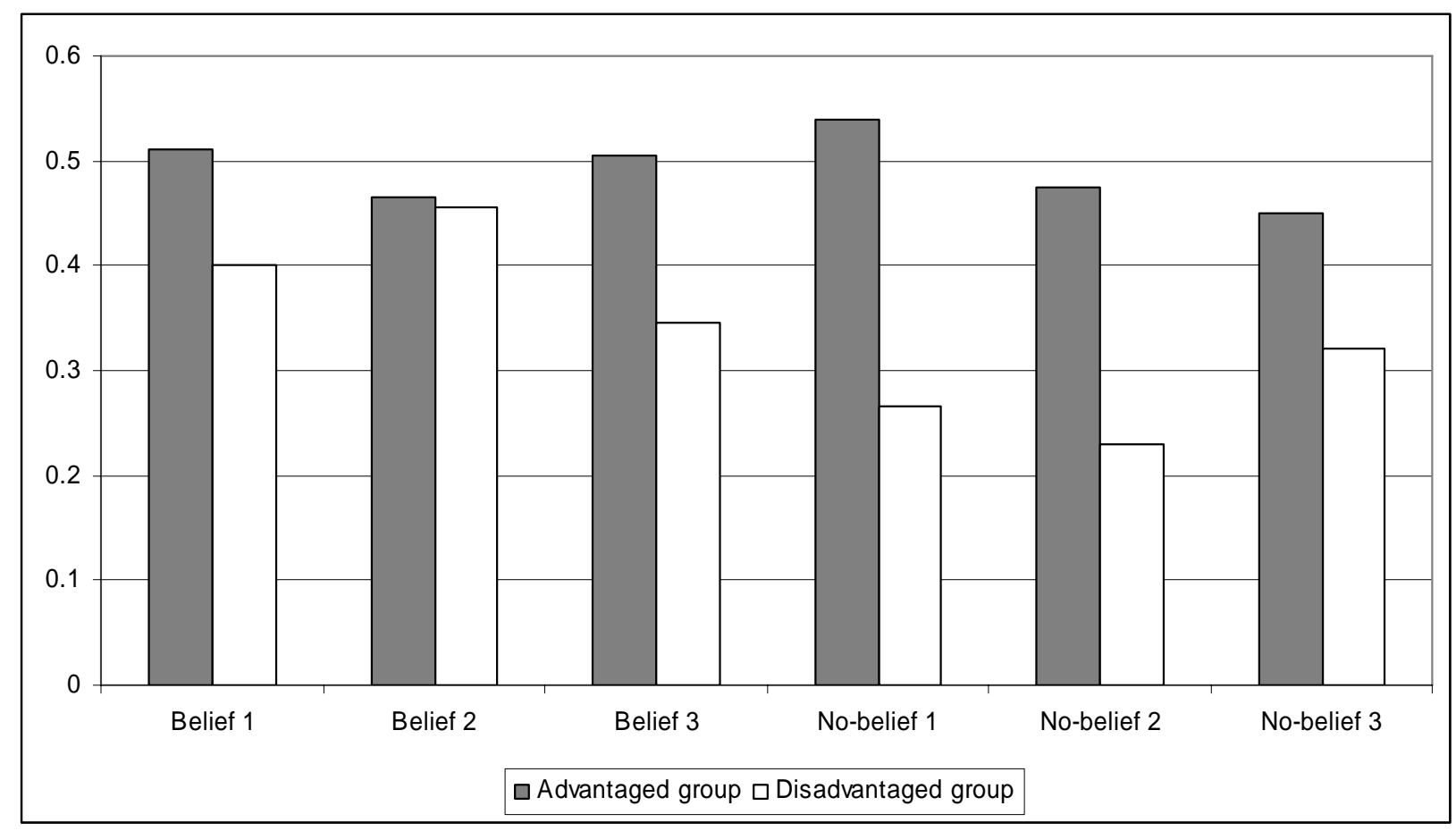


Table 1:Turnout Rates, 5-Round Averages, 3 Belief and 3 No Belief Sessions

\begin{tabular}{|r||r|r|r||r|r|r||r||}
\hline Rounds & Beliefs 1 & Beliefs 2 & Beliefs 3 & No Beliefs 1 & No Beliefs 2 & No Beliefs 3 & \multicolumn{2}{|c|}{$\begin{array}{l}\text { MW null hypo p- } \\
\text { value }\end{array}$} \\
\hline $1-5$ & 0.49 & 0.50 & 0.47 & 0.53 & 0.40 & 0.42 & 0.80 \\
\hline $6-10$ & 0.51 & 0.51 & 0.42 & 0.39 & 0.47 & 0.41 & 0.10 \\
\hline $11-15$ & 0.47 & 0.42 & 0.40 & 0.38 & 0.26 & 0.44 & 0.20 \\
\hline $16-20$ & 0.35 & 0.41 & 0.41 & 0.31 & 0.28 & 0.27 & 0.05 \\
\hline
\end{tabular}


Table 2: Probit analysis of the probability of being decisive, belief elicitation treatment

\begin{tabular}{|c|c|c|c|c|}
\hline & Model 1 & Model 2 & Model 3 & Model 4 \\
\hline & $\mathrm{b}(\mathrm{SE})$ & $\mathrm{b}(\mathrm{SE})$ & $\mathrm{b}(\mathrm{SE})$ & $\mathrm{b}(\mathrm{SE})$ \\
\hline Subjective & $.518 * *$ & & $.544^{* *}$ & \\
\hline $\operatorname{Pr}($ Decisive $)$ & $(.138)$ & & $(.140)$ & \\
\hline Subjective & & $.157 * *$ & & $.164 * *$ \\
\hline $\operatorname{Pr}($ Decisive $)>.18$ & & $(.067)$ & & $(.068)$ \\
\hline Actual frequency of & & & -.472 & -.357 \\
\hline decisiveness & & & $(.375)$ & $(.372)$ \\
\hline \multirow[t]{2}{*}{ Group wins tie } & $.250 * *$ & $.252 * *$ & $.249 * *$ & $.252 * *$ \\
\hline & $(.087)$ & $(.087)$ & $(.087)$ & $(.087)$ \\
\hline \multirow[t]{2}{*}{ Decisive $t-1$} & .108 & .150 & .188 & .212 \\
\hline & $(.119)$ & $(.118)$ & $(.135)$ & $(.134)$ \\
\hline \multirow[t]{2}{*}{ Participate $t-1$} & $.832 * *$ & $.805^{* *}$ & $.831 * *$ & $.803 * *$ \\
\hline & $(.083)$ & $(.082)$ & $(.083)$ & $(.083)$ \\
\hline \multirow{2}{*}{ Win $t-1$} & -.001 & -.005 & -.006 & -.008 \\
\hline & $(.099)$ & $(.099)$ & $(.099)$ & $(.099)$ \\
\hline Number of group & $-.097 * *$ & $-.093 * *$ & $-.096 * *$ & $-.092 * *$ \\
\hline tokens $t-1$ & $(.032)$ & $(.032)$ & $(.032)$ & $(.032)$ \\
\hline \multirow[t]{2}{*}{ Earnings $t-1$} & .050 & .026 & .039 & .016 \\
\hline & $(.087)$ & $(.087)$ & $(.088)$ & $(.087)$ \\
\hline \multirow[t]{2}{*}{ Tie $t-1$} & -.117 & -.098 & -.129 & -.106 \\
\hline & $(.120)$ & $(.120)$ & $(.120)$ & $(.120)$ \\
\hline \multirow[t]{2}{*}{ Session 1} & .078 & .093 & .111 & .119 \\
\hline & $(.098)$ & $(.098)$ & $(.102)$ & $(.102)$ \\
\hline \multirow[t]{2}{*}{ Session 2} & .056 & .081 & .088 & .105 \\
\hline & $(.096)$ & $(.096)$ & $(.100)$ & $(.099)$ \\
\hline \multirow[t]{2}{*}{ Period (trend) } & $-.014^{*}$ & $-.016^{*}$ & -.013 & $-.015^{*}$ \\
\hline & $(.007)$ & $(.007)$ & $(.007)$ & $(.057)$ \\
\hline$X^{2}$ & $132.3 * *$ & $123.56 * *$ & $133.88 * *$ & $124.48 * *$ \\
\hline Pseudo $R^{2}$ & .09 & .08 & .09 & .08 \\
\hline $\mathrm{N}$ & 1140 & 1140 & 1140 & 1140 \\
\hline
\end{tabular}

Note: Dependent variable is whether or not a token was bought. Table entries are probit coefficients with standard errors in parentheses, ${ }^{*} p \leq .05,{ }^{*} p \leq .01$ 
Table 3: Probit analysis of the probability of being decisive, control treatment and merged sample

\begin{tabular}{lll}
\hline & $\begin{array}{l}\text { Model 5: } \\
\text { Control }\end{array}$ & $\begin{array}{l}\text { Model 6: } \\
\text { Merged }\end{array}$ \\
\hline & $\mathrm{b}(\mathrm{SE})$ & $\mathrm{b}(\mathrm{SE})$ \\
\hline Actual pr(Decisive) & 1.219 & .056 \\
& $(.673)$ & $(.324)$ \\
Group wins tie & $.549^{* *}$ & $.386^{* * *}$ \\
& $(.082)$ & $(.059)$ \\
Decisive $t-1$ & .218 & $.247^{* * *}$ \\
& $(.166)$ & $(.104)$ \\
Participate $t-1$ & $.861^{* *}$ & $.814^{* * *}$ \\
& $(.092)$ & $(.061)$ \\
Win $t-1^{\#}$ & & .091 \\
& & $(.078)$ \\
Number of group tokens $t-1$ & $-.112^{* *}$ & $-.099^{* * *}$ \\
& $(.028)$ & $(.021)$ \\
Earnings $t-1$ & $.322^{* *}$ & .091 \\
& $(.110)$ & $(.070)$ \\
Tie $t-1$ & $.581^{* *}$ & .079 \\
& $(.193)$ & $(.103)$ \\
Beliefs elicited & No & .113 \\
& & $(.099)$ \\
Session 1 & .147 & .083 \\
Session 2 & $(.116)$ & $(.099)$ \\
& -.071 & -.081 \\
Session 4 & $(.102)$ & $(.096)$ \\
Session 5 & & .100 \\
& & $(.099)$ \\
Period (trend) & .093 \\
$X^{2}$ & & $(.101)$ \\
Pseudo $R^{2}$ & $(.008)$ & $(.005)$ \\
$\mathrm{N}$ & $187.67^{* *}$ & $265.13^{* * *}$ \\
& .12 & .1 \\
& 1140 & 2280 \\
\hline
\end{tabular}

Note: Dependent variable is whether or not a token was bought. Table entries are probit coefficients with standard errors in parentheses, ${ }^{*} p \leq .05,{ }^{* *} p \leq .01$

${ }^{\#}$ Win $t$ - 1 is dropped due to co-linearity. 